УДК 51-7

\title{
РАЗРАБОТКА МОДЕЛИ ВЫБОРА ОПТИМАЛЬНОГО ПАРАМЕТРА СЕНСОРА
}

\author{
Сараев Эмиль Камран оглы \\ студент \\ Курмангазиева Ляйля Таскалиевна \\ к.т.н., асс. профессор \\ Атырауский университет имени Х. Досмухамедова
}

\begin{abstract}
Аннотация В работе разработаны содержательная и математическая поставновка задачи выбора оптимального параметра сенсора для работы SIEM на основе многокритериального выбора. Разработана методика решения поставленной задачи, позволяющая снизить возможный ущерб от не предотвращения угроз безопасности.
\end{abstract}

Ключевые слова: сенсор, SIEM,алгоритм, многокритериальный выбор, безопасность.

\section{DEVELOPMENT OF A MODEL FOR CHOOSING THE OPTIMAL SENSOR PARAMETER}

\section{Sarayev Emil Kamran ogly Scientific adviser: Kurmangaziyeva Lyailya Taskalievna}

\begin{abstract}
The paper develops a meaningful and mathematical formulation of the problem of choosing the optimal sensor parameter for SIEM operation based on a multi-criteria selection. The method of solving the task has been developed, which allows to reduce the possible damage from not preventing security threats.
\end{abstract}

Key words: sensor, SIEM,algorithm, multi-criteria selection, security

\section{SIEM-системы}

\section{Решаемые задачи и принципы работы}

SecurityInformationEventManagement (SIEM) - Управление информацией и событиями в системе безопасности - общее название программных продуктов, ранее используемых по отдельности друг от друга, категории SIM (SecurityInformationManagement- управление информацией в системе 
безопасности) и SEM (SecurityEventManagement- управление событиями в системе безопасности) [1, с. 44].

Перед типовой системой SIEM ставятся следующие задачи.

- Консолидация и хранение журналов событий от различных источников - сетевых устройств, приложений, журналов ОС, средств защиты. Бывают ситуации, когда инцидент увидели поздно, а события уже давно затерты или журналы событий почему-либо недоступны, и причины инцидента выявить фактически невозможно. Кроме того, соединение с каждым источником и просмотр событий займет уйму времени.

- Предоставление инструментов для анализа событий и разбора инцидентов. Форматы событий в различных источниках различаются. Текстовый формат при больших объемах сильно утомляет, снижает вероятность выявления инцидента. Часть продуктов класса SIEM унифицирует события и делает их более читабельными, а интерфейс визуализирует только важные информационные события, акцентирует на них внимание, позволяет отфильтровывать некритические события.

- Корреляция и обработка по правилам. По одному событию не всегда можно судить об инциденте. Простейший пример - «loginfailed»: один случай ничего не значит, но три и более таких события с одной учетной записью уже могут свидетельствовать о попытках подбора. В простейшем случае в SIEM правила представлены в формате RBR (RuleBasedReasoning) и содержат набор условий, триггеры, счетчики, сценарий действий.

- Автоматическое оповещение и инцидент-менеджмент.

Основная задача SIEM - не просто собрать события, но автоматизировать процесс обнаружения инцидентов с документированием в собственном журнале или внешней системе HelpDesk, а также своевременно информировать о событии SIEM способна выявлять [2, с. 56].:

- сетевые атаки во внутреннем и внешнем периметрах;

- вирусные эпидемии или отдельные вирусные заражения, неудаленные вирусы, бэкдоры и трояны;

- попытки несанкционированного доступа к конфиденциальной информации;

- ошибки и сбои в работе информационных систем;

- уязвимости;

- ошибки конфигураций в средствах защиты и информационных системах. 
- Система SIEM универсальна за счет своей логики. Но для того чтобы возложенные на нее задачи решались - необходимы полезные источники и правила корреляции. Любое событие (например, если в определенной комнате открылась дверь) может быть подано на вход SIEM и использовано.

- Источники выбираются на основании следующих факторов:

- критичность системы (ценность, риски) и информации (обрабатываемой и хранимой);

- д достоверность и информативность источника событий;

- покрытие каналов передачи информации (должны учитываться не только внешний, но и внутренний периметр сети);

- решение спектра задач ИТ и ИБ (обеспечение непрерывности, расследование инцидентов, соблюдение политик, предотвращение утечек информации и т. п.).

Основные источники SIEM

- AccessControl, Authentication- для мониторинга контроля доступа к информационным системам и использования привилегий.

- Журналы событий серверов и рабочих станций - для контроля доступа, обеспечения непрерывности, соблюдения политик информационной безопасности.

- Сетевое активное оборудование (контроль изменений и доступ, счетчики сетевого трафика).

- IDSIIPS. События о сетевых атаках, изменение конфигураций и доступ к устройствам.

- Антивирусная защита. События о работоспособности ПО, базах данных, изменении конфигураций и политик, вредоносных программах.

- Сканеры уязвимостей. Инвентаризация активов, сервисов, программного обеспечения, уязвимостей, поставка инвентаризационных данных и топологической структуры.

- GRC-системы для учета рисков, критичности угрозы, приоритизации инцидента.

- Прочие системы защиты и контроля политик ИБ: DLP, антифрода, контроля устройств и т. п.

- Системы инвентаризации и asset-management. С целью контроля активов в инфраструктуре и выявления новых.

- Netflow и системы учета трафика. 
Решение SIEM включает в себя, как правило, несколько компонентов (рис. 1):

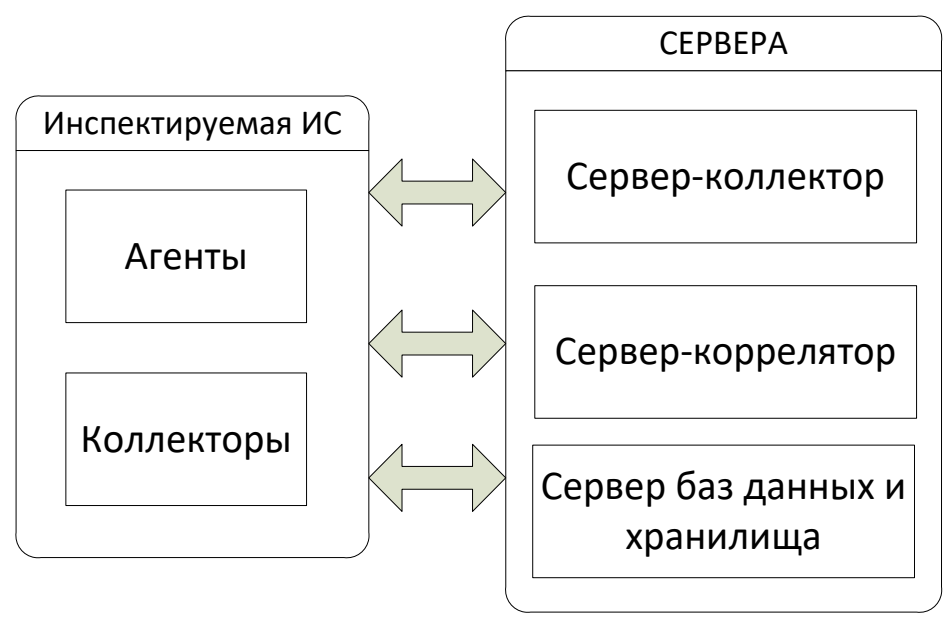

\section{Рис. 1. Структура SIEM}

- Агенты, инсталлируемые на рассматриваемую ИС (актуально для $\mathrm{OC}$; агент является некой отдельной программой (сервисом), которая локально проверяет все службы событий и отправляет статистику на сервер);

- Коллекторы агентов, по-другому - модули для понимания отдельного журнала всех события системы;

- Сервер-коллектор, необходимый для начального сбора событий из разных источников;

- Сервер-коррелятор, необходимый для сбора данных от коллекторов и агентов и изучения полученных данных по алгоритмам и правилам взаимного пересечения.

- Сервер БД и хранилища, которые несут ответственность за хранение всех данных [3, с. 528].

Зачастую, SIEM-система представлена в агентской архитектуре - месте для хранения информации - сервере программы, устанавливаемой поверх защищаемой ИТ структуры [4, с. 192].

\section{Математическая постановка задачи}

В рамках изучения различных систем: социальных, экономических, природных, техногенных, для учета всех факторов из работы как единого объекта, важно учитывать совокупность внешних факторов. Но не так часто связи между частями можно учесть из-за нехватки всего объема данных, а для некоторых задач - прогнозирования и имитации, данные вообще могут 
отсутствовать. Тогда нужные связи создаются путем оценок эксперта. Часто их реализуют в виде весовых параметров, применяемых для числовой оценки вклада текущего фактора в конечный результат[5, с. 25-35].

Учет весовых параметров. Для данного учета используются разные подходы, в рамках которых уже реализовано много методик. Т.к. нет задачи обобщенного описания используемых вариантов выражения весовых коэффициентов, выполнил лишь анализ главных подходов.

Прямая расстановка. Эксперты уточняют веса факторов, базируясь на требованиях, к примеру, чтобы сумма весов была от 1\% до 100\%, хотя часто может иметь место другая постоянная, если это удобно для дальнейших расчетов. Часто данный процесс путают с передачей факторам некоторых значений по явной числовой шкале, но в таком случае лучше назвать такие факторы показателями значимости, а не весами, т.к. тогда имеют сравнительную оценку, а не их влияние совокупный итог. Так или иначе, подобным способом реализуют и весовые коэффициенты, но тут мы закончим.

Трудности данного подхода заключены в возможности неявно держать в отдельных рамках все без исключения факторы, т.к., выделяя некое числовое значение любому фактору, эксперт должен при этом соотнести его с остальными. Сложность повышается в прогрессии с увеличением этих факторов.

Имеются и технические затруднения в работе специалиста, связанные с важностью периодического контроля текущей суммы весовых коэффициентов, чтобы не прийти к повышению указанной постоянной или передать крайним факторам оставшуюся большую часть. Если такое происходит, то принято пересчитать все отправленные коэффициенты, что можно сделать несколько раз, пока процесс обмена будет проходить. Число операций увеличивается с ростом числа факторов.

Ранжирование факторов. Такой подход позволяет облегчить экспертам работу, т.к. не нуждается в контроле общей суммы коэффициентов. В таком случае от экспертов необходимо ранжирование, т. е. упорядочивание рассматриваемых факторов, формирующих объект, по степени выявления их свойств в порядке их минимизации или роста.

$$
\left.\begin{array}{l}
R_{11}, R_{21}, \ldots, R_{i 1} \\
R_{12}, R_{22}, \ldots, R_{i 2} \\
\ldots \ldots \ldots \ldots \ldots \ldots \\
R_{1 j}, R_{2 j}, \ldots, R_{i j}
\end{array}\right\},
$$


где $\mathrm{R}_{\mathrm{ij}}$ - ранг (место), данный фактору $\mathrm{O}_{\mathrm{ij}}$-м экспертом в ряду из nизученных объектов, поставленным этим экспертом по степени выражения анализируемого свойства. Возможно, два и более фактора имеют одинаковый ранг, но тогда он имеет вид дроби. Сводные оценки весовых коэффициентов получаются по итогу усреднения частных рангов по столбцам n [6, c. 336].

Плюсы подобного метода состоят в его простоте, но это не всегда тот случай, когда простота влияет положительно, поскольку усреднение рангов приводит к более грубым оценкам весовых коэффициентов в сравнении с остальными методиками. Также он не избавляет эксперта от обязанности контроля всех факторов, как и в случае прямой расстановки.

Передача коэффициентов факторам. В этом методе экспертам предлагается провести оценку факторов по некоторой балльной шкале, к примеру, от 1 до 10. В итоге получаем:

$$
\left.\begin{array}{c}
y_{11}, y_{21}, \ldots, y_{i 1} \\
y_{12}, y_{22}, \ldots, y_{i 2} \\
\ldots \ldots \ldots \ldots \ldots \ldots \\
y_{1 j}, y_{2 j}, \ldots, y_{i j}
\end{array}\right\},
$$

где $\mathrm{y}_{\mathrm{ij}}$ - балльная оценка фактора, переданная от $\mathrm{j}$-го эксперта, $\mathrm{n}$ - сумма факторов, $\mathrm{m}-$ количество экспертов.

Сводные оценки весовых коэффициентов находят зачатую методом подбора соответствующей регрессионной модели. Среднюю оценку $\mathrm{w}_{\mathrm{i}}$ весовых коэффициентов факторов получают по тривиальным формулам:

$$
w_{i}=\frac{\sum_{j=1}^{m} w_{i j}}{\sum_{i=1}^{n} \sum_{j=1}^{m} w_{i j}},
$$

где $\mathrm{w}_{\mathrm{ij}}-$ вес i-го объекта, исходящий из оценок всех экспертов;

$$
w_{i j}=\frac{x_{i j}}{\sum_{i=1}^{n} x_{i j}},
$$

где $\mathrm{x}_{\mathrm{ij}}-$ оценка фактора $\mathrm{i}$, приведенная экспертом $\mathrm{j}$;

$\mathrm{n}$-число факторов, $\mathrm{m}$ - количество экспертов.

Такой метод в какой-то степени делает более слабой зависимость оценки отдельного фактора от остальных, но не полностью избавляет от нее, т.к. сопоставлять факторы необходимо, иначе коэффициенты значимости не будет возможности расставить корректно.

Любое из указанных суждений кодируется в диапазоне чисел от 1/9 до 9. [7, с.77-82].

Вычисление весов реализуется разными способами. Одним из доступных методов аппроксимации вектора весов служит вычисление 
отдельного вектора матрицы парных сравнений, обычно соответствующему большему собственному числу. Подобные алгоритмы получения собственного вектора являются подробно изученными, и описание их находится либо в монографиях, либо в другой литературе.

Метод МАИ имеет собственные параметры выражения качества работы эксперта - индекс согласованности (ИС), дающий данные об уровне нарушения численной и порядковой логичности экспертных суждений. Контроль кардинальности состоит в учете конкретных числовых характеристик, отклонение от которых говорит о наличии ошибок в процессе передачи экспертных суждений. Поэтому, если созданы отдельные правила кодировки экспертных суждений, к примеру, от 0 до 1, то экспертные суждения не могут выходить за рамки указанных в этих правилах множеств значений, т. е. быть больше единицы или отрицательными. Расположение по порядку помогает понять логику обоснований эксперта. В случае, если эксперт считает, что фактор А лучше фактора Б, а фактор Б, в свою очередь, лучше фактора В, то при парном сравнении фактор В не может быть лучше фактора А, т. е. выполняется неравенство А > Б > В. Несогласованность является значимым ограничивающим фактором для изучения отдельных проблем[8, с. 45-55].

ИС вычисляется таким образом: совместно с матрицей парных сравнений есть мера оценки степени отклонения от нужного значения. ИС в каждой матрице для каждой иерархии оценивается приближенно, с применением формулы:

$$
\text { ИC }=\frac{\lambda-n}{n-1},
$$

где $\lambda$ - собственное число, $n$ - число сравниваемых факторов.

ИС сравнивается с величиной, полученной при случайном выборе количественных величин, которая трактуется как средняя. Средние согласованности (СC) для случайных матриц разного порядка приведены в таблице 1 , где $n$ - число факторов.(табл. 1). Средние согласованности (СС) для случайных матриц разного порядка (рис.2). 
Таблица 1

Средние согласованности (СС) для случайных матриц разного порядка

\begin{tabular}{|l|l|l|l|l|l|l|l|l|l|l|}
\hline$n$ & 1 & 2 & 3 & 4 & 5 & 6 & 7 & 8 & 9 & 10 \\
\hline CC & 0 & 0 & 0,58 & 0,90 & 1,12 & 1,24 & 1,32 & 1,41 & 1,45 & 1,49 \\
\hline
\end{tabular}

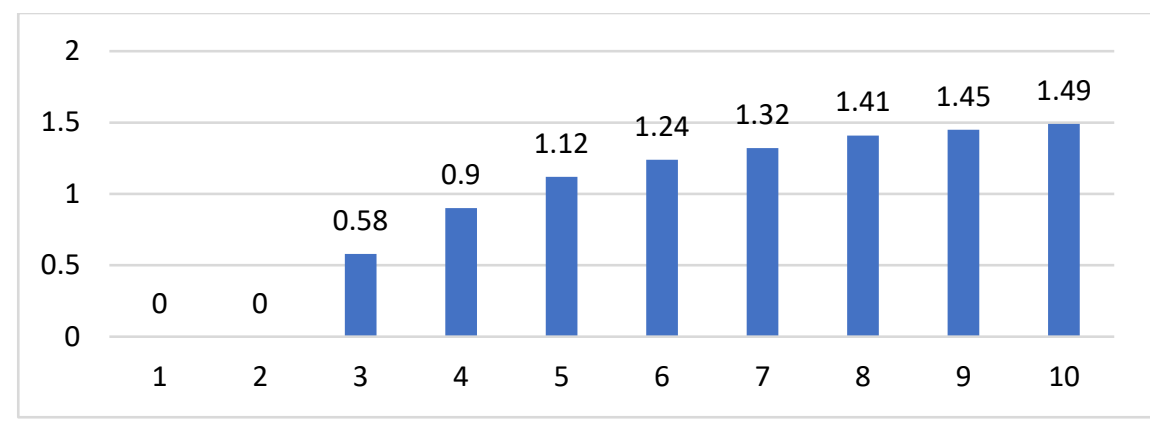

\section{Рис. 2. Средние согласованности (СС) для случайных матриц разного порядка}

Если разделить ИС на СС для матрицы того же порядка, то получим отношение согласованности (ОС):

$$
O C=\frac{h C}{C C} * 100 \%,
$$

Вначале кажется, что МАИ является оптимальным вариантом для решения множества различных задач, где методы экспертного анализа применяются как ключевые. Это в большинстве своем так и получается, и мы укажем основные причины этого.

Попарные сравнения. Попарные сравнения вещей можно найти и в природе человека. Минимальная необходимость всегда держать во внимании все факторы или, к примеру, часть некоторых факторов, позволяет эксперту уделять больше внимания отдельной проблеме: как фактор $\mathrm{A}_{\mathrm{j}}$ обгоняет фактор $\mathrm{B}_{\mathrm{j}}$ или отстает от него. Поэтому можно получать более точные итоги [9, c. 140-143].

Дополнительность начальной матрицы. В практике анализа системы часто возникают ситуации, когда число явных факторов корректируется. Связано это с периодичностью природных процессов, a также с корректировкой социально-экономических факторов. Тогда требуется добавлять, уменьшать или менять один фактор на другой. В рамках применения МАИ такое ведет к обязательности сравнения созданных пар или же к вычитанию строк и столбцов матрицы парных сравнений ранее убранных из анализа факторов, т. е. к реализации матричного минора. Все итоги 
прошлых опросов отражаются, и обновления всей анкеты, как это происходит в других вариантах, не требуется. Исходя из того, что процедура МАИ, часто ведет к поиску своего вектора требуемой матрицы, относящемуся к наибольшему собственному значению, со стороны технической реализации включение сторонних факторов считается повышением размерности отдельного линейного пространства из-за применения сторонних слагаемых.

Вербально-числовая шкала. Классические числовые шкалы зачастую не справляются со сравнением факторов, находящихся в разных величинах и сферах. Трудно сравнить факторы, итогами которых, изначально, приводятся качественные параметры, а затем - количественные. Зачастую используемая шкала Харрингтона имеет на входе лишь некоторые суммарные параметры, которые могут корректироваться в диапазоне от 0 до 1. Вербально-числовые шкалы, куда и относится шкала Саати, реализована для оценки таких нестыковок показателей базовых факторов.

Доступный критерий качества деятельности специалиста. По факту оценки, эксперты часто нуждаются в проверке. Чаще всего для этого применяются разные числовые параметры, реализованные для групповых и персональных опросов. При этом вопрос наилучшего критерия остается открытым, да и выбор его доступен. В этом смысле перенос в МАИ параметра отношения согласованности несет некие удобства, особенно хорошо это отражается в процессе реализации автоматического программного комплекса.

Недостатки методики: не все выигрышные стороны МАИ так явно видны. Есть ряд вопросов при анализе итогов, и связаны они, чаще всего, с оцениванием корректности работы эксперта - с уровнем согласованности.

Использование транзитивности для качественных параметров. Она может отлично справится в том случае, когда все параметры анализируемой системы выступают в качестве числовых величин. Но в случае, если это не так, транзитивность чаще всего переходит в борьбу с логикой исследователя.

«Обратная» логика. Процент качества работы эксперт, а также отношение согласованности основываются на корректировке явно определенной характеристики, например, математического ожидания. Как и любой критерий, имеющий стабильный характер, отношение согласованности формально и зачастую приводит к интерпретируемым итогам.

Опишем решение задачи выбора критериев и сравнения параметров сенсора для SIEM-системы при помощи методики анализа иерархий. Задача 
сравнительного анализа реализовывалась в разных проектах, но изучение самого перечня критериев было сделано не так часто.

На верхнем уровне иерархии стоит цель - выбор оптимального сравнения параметров сенсора для SIEM-системы. Ниже расположены критерии выбора. Эти критерии считаются неравнозначными. Под данными критериями находятся изучаемые методы определения и сравнения параметров сенсора для SIEM-системы.

Второй шаг включает в себя выделение веса важности Sij критериев. Это реализуется методом проверки всевозможных парных сравнений параметров на качественной шкале и анализа построенной матрицы парных сравнений.

На третьем шаге определяются приоритеты методов выбора и сравнения инвестиционных проектов $\mathrm{C}_{\mathrm{ij}}$ по отношению к каждому из 9 критериев. Для этого эксперт выполняет их всевозможные парные сравнения на качественной шкале. Для каждого из критериев $K_{t}$ путем обработки матрицы парных сравнений формируется вектор весовых коэффициентов $S\left(K_{t}\right)=\left\{S_{i}\left(K_{t}\right)\right\}, i=$ $\overline{1,9}$.

Объединив векторы весовых коэффициентов по каждому из критериев, мы получим полную матрицу приоритетов выбора методов выбора и сравнения инвестиционных проектов размерности 9x11.

На четвертом шаге определяется итоговый вектор $\mathrm{w}=\left(\mathrm{w}_{1}, \ldots, \mathrm{w}_{11}\right)$ приоритетов методов выбора и сравнения инвестиционных проектов.

Рассмотрим трехуровневую схему иерархии (Цель - критерии альтернативы). В более сложных случаях можно рассматривать и схему с большим числом уровней.

Для математической постановки введем в рассмотрение следующие множества:

1. $K=\left\{k_{1}, k_{2}, \ldots, k_{n}\right\}-$ множество критериев (или требований для задач выбора СЗИ), $N=\{1,2, \ldots, n\}$ - множество индексов критериев.

2. $A=\left\{a_{1}, a_{2}, \ldots, a_{m}\right\}$ - множество альтернатив (для задач выбора СЗИ, альтернатива это одно СЗИ), $M=\{1,2, \ldots, m\}-$ множество индексов альтернатив, соответственно.

Для элементов этих множеств заданы следующие параметры:

1. $v_{i}^{(k)}, \forall i \in N$ - «веса» или «важности» критериев с точки зрения достижения цели, определяются экспертами, на данные «веса» наложено условие нормировки: $\sum_{i \in N} v_{i}^{(k)}=1$. 
2. $v_{i j}^{(a)}, \forall i \in N, j \in M-$ «вес» («важность») $j$-ой альтернативы для достижения $i$-го критерия. На эти «веса» также наложены условия нормировки вида: $\sum_{j \in M} v_{i j}^{(a)}=1, \forall i \in N$.

Тогда глобальный приоритет $\mathrm{j}$ - ой альтернативы для достижения цели вычисляется следующим образом:

$$
F_{j}=\sum_{i \in N} v_{i}^{(k)} v_{i j}^{(a)}, \forall j \in M
$$

Постановка задачи выбора альтернативы с максимальным глобальным приоритетом имеет вид:

$$
F_{j}=\sum_{i \in N} v_{i}^{(k)} v_{i j}^{(a)} \rightarrow \max _{j \in M}
$$

Рассмотрим решение задачи многокритериального выбора критерия работоспособности сенсора для SIEM-системы с помощью метода анализа иерархий. Задача сравнительного анализа решалась в различных работах, однако исследований относительно перечня критериев производилось недостаточно.

В качестве критериев выбора оптимального параметра сенсора для SIEM-системы будем использовать следующие:

- $\mathrm{K} 1=«$ Нагрузка на систему $(\mathrm{OC}) » ;$

- $\mathrm{K} 2=\ll$ «ремя реакции»;

- $\quad \mathrm{K} 3=\ll$ Время работы»;

- $\mathrm{K} 4=$ «Результативность», то есть эффективность применяемых средств защиты в рассматриваемой ситуации;

- $\quad \mathrm{K} 5=«$ Стоимость реализации»;

- $\mathrm{K} 6=$ = «рудоемкость реализации»;

- $\mathrm{K} 7=\ll У$ ниверсальность»;

- $\mathrm{K} 8=«$ Качество реализации»;

- $\mathrm{K} 9=«$ Распространенность».

\section{Список литературы}

1. Miller D. R., Harris Sh., Harper A., VanDyke S., Blask C. Security information and event management (SIEM) implementation. NewYork: McGrawHill.- 2011.- 464 c.

2. И.В. Котенко, И.Б. Саенко, О.В. Полубелова, А.А. Чечулин, Применение технологии управления информацией и событиями безопасности для защиты информации в критически важных инфраструктурах, Тр. СПИИРАН, 2012, выпуск- 20. 27-56 // URL:http://www.mathnet.ru/links/ 
c5b9b0585c8b0c2acaeb5af09cc29a41/trspy488.pdf.

3. Арнольд Виллемер: Программирование на С++. М.: Издательство: Эксмо,- 2013.- 528 с.

4. Болтян А.В. Прогнозирование и оценка параметров продукции / А.В. Болтян, И.А. Горобец. Донецк: Норд-Пресс,- 2004. -192 с.

5. Бычков Е.Д., Кладов В. В. Защита Web-сервера от атак типа DDOS на основе модели нечеткого вывода II Актуальные проблемы гуманитарных и естественных наук.- 2013.- №5.-25-35с.

6. В. Тимофеев: Самоучитель С++ как он есть. М.: Издательство: Бином, -2009. -336 c.

7. Вехов В.Б. Возможности правоохранительных органов по противодействию DDoS-атакам / В.Б. Вехов // Защита информации. Инсайд. 2008. -№ 3. -C. 77-82.

8. Гольдштейн A. DDoS-атаки: механизмы создания и варианты защиты // InformationSecurity / Информационная безопасность. 2012.- №2.-45$55 \mathrm{c}$.

9. Жилина Е.В., Ружицкий А.Г. Принципы сетевого сканирования в пентестинге // Fundamental and applied science today V. Vol. 1. Proceedings of the Conference (Материалы V международной научно-практической конференции «Фундаментальные и прикладные науки сегодня» 30-31 марта 2015 г.). NorthCharleston, USA. -2015. - T. 1. C. 140-143.

() Э.К.Сараев, Л.Т. Курмангазиева, 2021 